DOI: http://doi.org/10.22585/hospdomic.v5i2.136

\title{
Valoración geriátrica integral
}

\section{Comprehensive Geriatric Assessment}

\author{
Carmina Wanden-Berghe ${ }^{1,2}$ (D) 0000-0002-6871-5737 \\ 1. Instituto de Investigación Sanitaria y Biomédica de Alicante (ISABIAL-FISABIO). Alicante. España. \\ 2. Hospital General Universitario de Alicante. Unidad de Hospitalización a Domicilio. Alicante. España.
}

Correspondencia/Correspondence

Carmina Wanden-Berghe

carminaw@telefonica.net

Recibido/Received

10.04.2021

Aceptado/Accepted

12.04 .2021

Conflicto de Intereses/Competing interest

La autora declara la inexistencia de conflicto de interés
Financiación/Funding

Sin financiación

Contribuciones de autoría/Author contributions

Autora única

Agradecimientos/Acknowledgments

Sin agradecimientos

\section{CÓMO CITAR ESTE TRABAJO | HOW TO CITE THIS PAPER}

Wanden-Berghe C. Valoración geriátrica integral. Hosp Domic. 2021;5(2): 1 15-24. 


\section{RESUMEN}

El objetivo principal de la valoración geriátrica integral es diseñar un plan individualizado preventivo, terapéutico y rehabilitador, con el fin de lograr el mayor nivel de independencia y calidad de vida del anciano. Se puede decir que tanto en el ámbito hospitalario como en el de atención primaria la valoración geriátrica integral es el mejor instrumento, el cual puede facilitar una práctica médica con una visión holística que garantice una atención adecuada, efectiva y de calidad.

Palabras clave: Geriatría; Salud del Anciano; Indicadores de Salud; Servicios de Salud para Ancianos.

\section{ABSTRACT}

The main objective of the comprehensive geriatric assessment is to design an individualized plan of preventive action, therapeutic or rehabilitation, in order to achieve the highest level of independence and quality of life for the elderly. It is said that both in the hospital setting as well as primary care the comprehensive geriatric assessment is the best instrument, which can facilitate a family medical practice with a holistic vision that ensures a proper, and effective care and quality.

Keywords: Geriatrics; Health of the Elderly; Health Status Indicators; Health Services for the Aged. 


\section{INTRODUCCIÓN}

Conforme avanza la edad y los sujetos envejecen, cobra mayor importancia mantener el equilibrio funcional de todos los órganos y sistemas para mantener un nivel óptimo de funcionalidad.

Las características inherentes a la edad geriátrica, en las que confluyen los aspectos intrínsecos del envejecimiento fisiológico y la especial forma de presentación de las enfermedades, hacen necesario un sistema específico de valoración. En esta población, la prevalencia de alteraciones no diagnósticas, de disfunciones potencialmente reversibles no identificadas, y que no son susceptibles de ponerse de manifiesto con una valoración clínica al uso, hace necesario un proceso diagnóstico dinámico y estructurado que permita detectar y cuantificar los problemas, necesidades y capacidades del anciano en las esferas clínica, funcional, mental y social, para elaborar una estrategia interdisciplinar de intervención, tratamiento y seguimiento a largo plazo con el fin de lograr el mayor grado de independencia y, en definitiva, calidad de vida ${ }^{(1,2)}$.

Cada uno de los cuatro pilares en los que se sustenta la valoración geriátrica y que contribuyen, de una forma decisiva, a mantener el equilibrio de salud y calidad de vida son: el clínico, el funcional, el mental y el social. Así, sería un gran fracaso recomendar el mejor de los tratamientos para cualquier dolencia en el anciano si no hemos valorado si la situación mental, funcional y social, va a permitir un buen cumplimiento terapéutico.

Los objetivos de la valoración geriátrica integral incluyen ${ }^{(3)}$.

- Mejorar la exactitud diagnóstica atendiendo a las cuatro esferas; clínica, funcional, mental y social.

- Descubrir problemas no diagnosticados previamente y potencialmente tratables.

- Establecer un tratamiento adecuado a las necesidades del anciano, contemplando las alteraciones identificadas en las cuatro esferas.

- Mejorar el estado funcional y cognitivo.

- Mejorar la calidad de vida.

- Conocer los recursos del paciente y su entorno sociofamiliar.

- Para situar al paciente en el nivel médico y social más adecuado a sus necesidades, fomentando en la medida que sea posible la autonomía, intentando evitar los ingresos hospitalarios y las institucionalizaciones.

- Disminuir la mortalidad.

Los métodos que se utilizan para realizar la valoración geriátrica integral comprenden; la anamnesis, la exploración física y una serie de instrumentos específicos «escalas de valoración» que facilitan la detección, cuantificación y seguimiento de los problemas, además de servir para unificar la información y facilitar la comprensión y comunicación entre los diferentes profesionales ${ }^{(4)}$.

\section{EXPLORACIÓN DEL ÁREA CLIINICA}

Siempre hay que comenzar con una anamnesis detallada para recopilar toda la historia de posibles enfermedades que haya tenido el paciente a lo largo de su vida ya que esto nos ayudará a comprender posibles secuelas funcionales, así como habrá que recoger minuciosamente los hábitos de vida, los tratamientos vigentes, ya sean farmacológicos o de cualquier otro tipo, y la adherencia que tengan a los mismos. No siempre es fácil ya que en muchas ocasiones nos tropezamos con serias dificultades de comunicación que pueden ser salvadas mediante el apoyo de un familiar o 
cuidador. También es frecuente que el anciano refiera múltiples quejas que habitualmente suele dar énfasis a los problemas más crónicos sin reparar apenas o vagamente en el motivo que le ha traído a la consulta o al hospital.

Debe incluir un interrogatorio dirigido a detectar la presencia, ya sea instaurada o incipiente, de alguno de los síndromes geriátricos (inmovilidad, caídas, malnutrición, deterioro cognitivo, depresión, disminución auditiva o de agudeza visual, estreñimiento, incontinencia, ...).

La historia nutricional debe contemplar las posibles alteraciones que presente para una adecuada alimentación como son: alteraciones en la masticación, deglución, xerostomía, utilización de sondas, etc. También, se tiene que recopilar, no solo la cantidad de alimentos que consume diariamente y la cuantificación calórica y proteica, además debe informar sobre la calidad de los alimentos, ¿están adecuadamente representados en la dieta los diferentes grupos de alimentos?, ¿la distribución horaria es adecuada para el sujeto valorado y el reparto calórico está en consonancia con sus necesidades?, ¿los alimentos están adaptados a las posibles alteraciones que presenta (masticación, deglución, anorexia, diabetes, enfermedad renal crónica, otras patologías)?, ¿está utilizando espesantes, suplementos nutricionales, algún modulo?

No olvidaremos la importancia de evaluar los factores que pueden llegar a afectar al estado nutricional como problemas funcionales que afecten a la independencia para comer y a la capacidad para adquisición y preparación de los alimentos, cambios anatómicos, problemas psicoafectivos, problemas económicos, etc. Es necesario, identificar a los pacientes en riesgo de desnutrición o aquellos que ya estén desnutridos mediante cuestionarios de cribado. De los validados en la población geriátrica, los más conocidos son el Mini Nutritional Assessment (MNA) ${ }^{(5)}$ y la aplicación (App) Herramienta de Evaluación Nutricional $(\mathrm{HEN})^{(6)}$. Y, posteriormente con una evaluación nutricional completa para tratar nutricionalmente el problema que puedan presentar estos pacientes.

La exploración física tiene que ir "más allá" de lo que al sujeto le preocupa, o nos refiere, ya que por la particular forma de presentación de las patologías en el anciano algunas situaciones nos deben hacer sospechar. Es frecuente un proceso infeccioso sin fiebre y tan solo con un estado de estupor o adormecimiento, o un infarto de miocardio indoloro, etc. Aunque, la técnica exploratoria no difiere de la utilizada en cualquier otra población, mediante la inspección, palpación, percusión y auscultación, es más minuciosa y laboriosa por la cantidad de hallazgos que muy probablemente se van a encontrar y el relacionarlos con algún diagnostico potencialmente tratable.

La exploración física la complementaremos con aquellas pruebas de laboratorio o de imagen que consideremos necesarias para llegar a un diagnóstico. Es fundamental tener siempre presente si la información que vamos a obtener va a servirnos para tomar decisiones que sean aplicables al paciente, valorando siempre la posible iatrogenia de las pruebas, y si existe posibilidad terapéutica de la patología buscada en función de la situación funcional y mental, evitando el encarnizamiento tanto diagnóstico como terapéutico ${ }^{(7)}$.

\section{EXPLORACIÓN DEL ÁREA FUNCIONAL}

El área funcional está formada por el conjunto de capacidades del anciano para realizar las actividades de su vida, su actividad habitual, y mantener su independencia en el medio en que se encuentra.

Las actividades de la vida diaria se clasifican en actividades básicas de la vida diaria (ABVD), actividades instrumentales de la vida diaria (AIVD) y actividades avanzadas de la vida diaria (AAVD) En la tabla 1, se exponen las características y los métodos de evaluación ${ }^{(8,9)}$. Existen muchas esca- 
las y cada profesional o centro asistencial deberán seleccionar la que más se adapte a su forma de trabajar, utilizando siempre la misma para poder comparar resultados evolutivos intra y entre pacientes.

Tabla 1. Valoración funcional.

\begin{tabular}{|c|c|c|c|}
\hline Actividades & Capacidades & Escalas & Items \\
\hline \multirow[t]{2}{*}{ ABVD } & \multirow[t]{2}{*}{$\begin{array}{l}\text { Tareas diarias para el } \\
\text { autocuidado } \\
\text { (aseo, vestido, alimentación...) }\end{array}$} & Índice de KATZ & $\begin{array}{l}\text { Baño. } \\
\text { Vestirse/desvestirse. } \\
\text { Uso del retrete. } \\
\text { Movilidad. } \\
\text { Continencia. } \\
\text { Alimentación. }\end{array}$ \\
\hline & & Índice de Barthel. & $\begin{array}{l}\text { Baño. } \\
\text { Vestido. } \\
\text { Aseo personal. } \\
\text { Uso del retrete. } \\
\text { Transferencias: (traslado } \\
\text { cama-sillón). } \\
\text { Subir/bajar escalones. } \\
\text { Continencia urinaria. } \\
\text { Continencia fecal. } \\
\text { Alimentación. }\end{array}$ \\
\hline AIVD & $\begin{array}{l}\text { Interacción con } \\
\text { el medio para } \\
\text { mantener su } \\
\text { independencia } \\
\text { (cocinar, comprar, } \\
\text { uso del teléfono, } \\
\text { cuenta bancaria...) }\end{array}$ & $\begin{array}{l}\text { Índice de Lawton y } \\
\text { Brody }\end{array}$ & $\begin{array}{l}\text { Usar el teléfono. } \\
\text { Ir de compras. } \\
\text { Preparar la comida. } \\
\text { Realizar tareas del hogar. } \\
\text { Lavar la ropa. } \\
\text { Utilizar transportes. } \\
\text { Controlar la medicación. } \\
\text { Manejar el dinero. }\end{array}$ \\
\hline AAVD & $\begin{array}{l}\text { Participación } \\
\text { en actividades } \\
\text { sociales, } \\
\text { actividades } \\
\text { recreativas, trabajo, } \\
\text { viajes y ejercicio } \\
\text { físico intenso. }\end{array}$ & $\begin{array}{l}\text { Índice de Lawton y } \\
\text { Brody }\end{array}$ & \\
\hline \multicolumn{4}{|c|}{$\begin{array}{l}\text { Actividades básicas de la vida diaria (ABVD) } \\
\text { Actividades instrumentales de la vida diaria (AIVD) } \\
\text { Actividades avanzadas de la vida diaria (AAVD) }\end{array}$} \\
\hline
\end{tabular}


Además de estos cuestionarios, es de gran utilidad la exploración objetiva. Una medición objetiva del funcionamiento físico y de la capacidad de realizar movimiento o actividades específicas. Para ello, se valora la capacidad de realizar series de repeticiones de movimientos o cronometrar el tiempo de ejecución de una actividad concreta. La Organización Mundial de la Salud cataloga a las personas como válidas, con deficiencia, discapacidad o minusvalía en función de los resultados obtenidos.

Una de las baterías exploratorias más utilizada en la práctica clínica es la Short Physical Performance Battery (SPPB), ver figura 1. Consiste en la realización de tres pruebas:

- Equilibrio (en tres posiciones: pies juntos, semi-tándem y tándem).

- Velocidad de la marcha (sobre 4 metros).

- Levantarse y sentarse en una silla cinco veces.

Figura 1. Short Physical Performance Battery (SPPB)

\section{PUNTUACIÓN SPPB:}

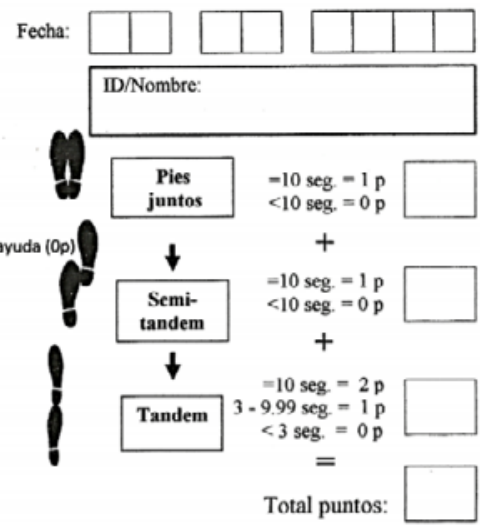

\section{Puntuación caminar}

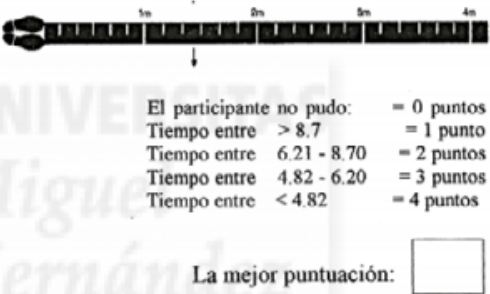

Si el participante ha fallado o no lo ha intentado,

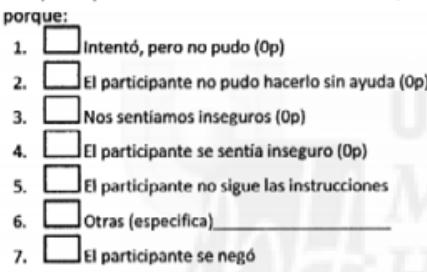

\section{Puntuación levantarse/sentarse x5}

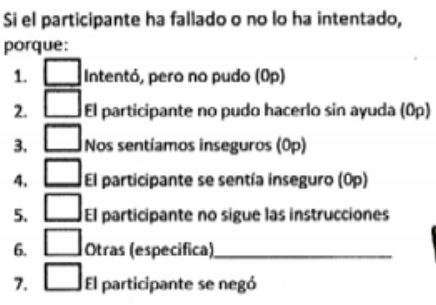

El participante no pudo $>60 \mathrm{seg}=0$ puntos Tiempo entre $\geq 16.7 \mathrm{seg}=1$ punto Tiempo entre $13.7-16.69 \mathrm{seg}=2$ puntos Tiempo entre $11.20-13.69 \mathrm{seg}=3$ puntos Tiempo entre $\leq 11.19 \mathrm{seg}=4$ puntos

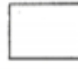


Es muy importante respetar la secuencia de las pruebas, ya que, si empezamos por las levantadas, el paciente se puede fatigar y ofrecer rendimientos falsamente bajos en las otros dos. El tiempo medio de administración, con entrenamiento, se sitúa entre los 6 y los 10 minutos.

La puntuación y valoración del resultado total del SPPB resulta de la suma de los tres sub-tests, y oscila entre 0 y 12 puntos, mientras más baja es la puntuación menor capacidad, cambios en 1 punto tienen significado clínico. Una puntuación por debajo de 10 indica fragilidad y un elevado riesgo de discapacidad, así como de caídas ${ }^{(10)}$.

A medida que el grado de deterioro funcional progresa, aumentan el riesgo de mortalidad, el número de ingresos hospitalarios en un año, y la estancia media en días de ingreso, la frecuentación de las consultas médicas y de los servicios de urgencias, el consumo de fármacos, el riesgo de institucionalización y la necesidad de recursos sociales.

\section{VALORACIÓN DEL ÁREA MENTAL}

Exploraremos la parte cognitiva y la parte afectiva ya que ambas componen la salud mental.

La parte cognitiva depende de entre otros, factores orgánicos, psicosociales, y de las enfermedades que comprenden la biografía del sujeto (hipertensión, diabetes, alteraciones tiroideas, insuficiencia renal o hepática, etc.) polifarmacia y alteraciones en los órganos de los sentidos. Todos estos factores también se correlacionan con la parte afectiva, con las posibles distimias que se puedan presentar, de las que los trastornos por ansiedad y depresión son los más frecuentes en este grupo de edad. Por lo tanto, la identificación de estos factores de riesgo y otros asociados, podría ser una forma de identificar al anciano con fragilidad afectiva.

La prevalencia de problemas en el área mental es muy grande, alrededor del $25 \%$ de los ancianos tienen algún trastorno psiquiátrico. La depresión es el más frecuente, presente $20 \%$ de los hombres y hasta en $40 \%$ de las mujeres, y tiene importantes repercusiones en la calidad de vida. Muchas veces en la entrevista clínica ya podemos detectarla y además disponemos de diferentes pruebas, la más empleada es la Escala de Depresión de Yesavage(11), en sus dos versiones la larga con 30 ítems y la corta de 15 ítems, que es la más recomendada para realizar el cribado, una puntuación de 0 a 5 puntos indica normalidad; entre 6 y 9, probable depresión, y más de 10, depresión. Otra escala muy utilizada por su facilidad de realización es la de depresión y ansiedad de Goldberg, breve y sencilla, tiene la finalidad de lograr una entrevista de cribaje de estos dos trastornos psicopatológicos, la ansiedad y la depresión.

Las demencias afectan en España al 1,07\% de las personas entre los 65-69 años; 3,4\% en los 70-74 años; $6,9 \%$ en los 75-79 años; $12,1 \%$ en los 80-84; 20,1\% en los 85-89; y 39,2\% entre los mayores de 90 años. "Demencia" es un término genérico para referirse a varias enfermedades, en su mayoría progresivas, que afectan a la memoria, a otras capacidades cognitivas y al comportamiento, y que interfieren notablemente en la capacidad de la persona para llevar a cabo las actividades cotidianas. La forma más común de demencia es la enfermedad de Alzheimer, que puede representar entre un $60 \%$ y un $70 \%$ de los casos. Otras formas frecuentes son la demencia vascular, la demencia por cuerpos de Lewy y un grupo de enfermedades que contribuyen a la demencia frontotemporal. Los límites entre las distintas formas de demencia son difusos y frecuentemente coexisten formas mixtas ${ }^{(12)}$.

La prueba para valorar la esfera cognitiva más utilizada es el Mini Mental State Examination de Folstein (MMSE), que es una prueba de cribado que valora la orientación temporo-espacial, la memoria reciente y la fijación de la misma, la atención, cálculo, capacidad de abstracción lenguaje 
y praxis $^{(13)}$. Debe ajustarse en función de la escolaridad, puntuaciones por debajo de 24 indican deterioro cognitivo (ver figura 2).

Figura 2. Mini Mental State Examination de Folstein (MMSE)

\begin{tabular}{|l|c|}
\hline ORIENTACIÓN & PUNTOS \\
\hline ¿Qué año-estación-fecha-dia-mes es? & $(5)$ \\
\hline ¿Dónde estamos? (estado-pais-cuidad-hospital-piso) & $(5)$ \\
\hline MEMORIA INMEDIATA & \\
\hline $\begin{array}{l}\text { Repetir } 3 \text { nombres ("mesa", "llave", "libro"). Repetirlos de nuevo hasta que aprenda } \\
\text { los tres nombres y anotar el número de ensayos. }\end{array}$ & $(3)$ \\
\hline & \\
\hline ATENCIÓN Y CÁLCULO & (3) \\
\hline $\begin{array}{l}\text { Restar 7 a partir de 100, 5 veces consecutivas. Como alternativa, deletrear "mundo" } \\
\text { al revés. }\end{array}$ & \\
\hline & \\
\hline RECUERDO DIFERIDO total: & $(1)$ \\
\hline Repetir los 3 nombres aprendidos antes. & $(3)$ \\
\hline & $(1)$ \\
\hline LENGUAJE Y CONSTRUCCIÓN & $(3)$ \\
\hline Nombrar un lápiz y un reloj mostrados & \\
\hline Repetir la frase "Ni sí es, ni no es, ni peros" & \\
\hline $\begin{array}{l}\text { Realizar correctamente las tres órdenes siguientes: "Tome este papel con la mano } \\
\text { derecha, dóblelo por la mitad y póngalo en el suelo" }\end{array}$ & \\
\hline Leer y ejecutar la frase "Cierre los ojos" & \\
\hline Escribir una frase con sujeto y predicado & \\
\hline Copiar este dibujo: & \\
\hline
\end{tabular}




\section{ÁREA SOCIAL}

Debemos considerar algunos aspectos para conocer la relación del adulto mayor con su entorno. Las características sociales en las que vive pueden influir directamente en su estado de salud y sobre la evolución de sus patologías. Esto hace necesario conocer algunos aspectos; lugar donde vive, con quién vive, situación del cuidador principal, características de la vivienda (barreras arquitectónicas), nivel de ingresos, etcétera. Existen varias escalas para recoger esta información y evaluar si el entorno es adecuado o no, una de las más extendidas en su uso es la escala sociofamiliar de Gijón ${ }^{(14)}$.

Los aspectos relacionados con el hogar y la familia deben considerarse en el plan de cuidados y atención. Una vez identificados, si fuera necesario derivaríamos para evaluación e intervención de la trabajadora social.

Cuando exista necesidad de cuidados, es importante identificar al cuidador, y si este está en condiciones para prestar el apoyo que se necesita, la sobrecarga que soportan en muchas ocasiones los cuidadores, tanto física como afectiva pueden llevar a una claudicación. Identificar esta sobrecarga es importante para que se tomen las medidas para corregirla o evitarla y poder continuar con el plan de cuidados. La escala de Zarit nos ayuda a identificar estos casos, mide el grado en que el cuidador percibe que su trabajo de asistencia al enfermo altera su propia salud física y emocional, así como su situación económica. Es un instrumento autoadministrado del que existen varias versiones, la más extendida consta de 22 ítems ${ }^{(15)}$.

Una vez realizada la valoración geriátrica integral es necesario y de gran utilidad elaborar un listado de problemas, priorizándolos y estableciendo un plan realista de intervención terapéutica y de cuidados.

\section{BIBLIOGRAFÍA}

1. Rubenstein LZ. Geriatric assessment: an overview of its impacts. Clin Geriatr Med. 1987;3(1):115. DOI: 10.1016/S0749-0690(18)30823-1

2. Kane RA, Bayer AJ. Assessment of functional status. In: Pathy MSJ, editor. Principles and practice of geriatric medicine. 2nd ed. Chichester: John Wiley \& Sons; 1991. p 265-71.

3. González Montalvo Jl. Principios básicos de la valoración geriátrica integral. En: Valoración Geriátrica Integral. Barcelona: Glosa Ediciones; 2001. p 15-27.

4. Sanjoaquín Romero AC, Fernández Arín E, Mesa Lampré MP, García-Arilla Calvo E. Valoración geriátrica Integral. En: Tratado de geriatría para residentes. Madrid: Sociedad Española de Geriatría y Gerontología; 2006. p 59-69.

5. Guigoz Y, Vellas B, Garry PJ. Assessing the nutritional status of the elderly:

6. The Mini Nutritional Assessment as part of the geriatric evaluation. Nutr Rev.

7. 1996;54 (1 Pt 2):S59-65. DOI: 10.1111/j.1753-4887.1996.tb03793.x.

8. Wanden-Berghe C, Alvarez Sabucedo LM. Alonso-Rorís VM, Desarrollo de una Aplicación Móvil para la Evaluación Nutricional. Hosp Domic. 2019;3(2):139-47. DOI: 10.22585/hospdomic. v3i2.71

9. Gómez Pavón J. ¿Es ético limitar el acceso de determinados tratamientos por motivos de edad? JANO. 2003;64(1474):31-4. 
10. Katz S, Ford AB, Moskowitz RW, Jackson BA, Jaffe MW. Studies of illness in the age: The index of ADL, a standarized measure of biological and psychosocial function. JAMA.1963;185(12):9149. DOI: 10.1001/jama.1963.03060120024016

11. Lawton MP, Brody EM. Assessment of older people: selfmaintaining and instrumental activities of daily living. Gerontologist. 1969;9(3):179-86.

12. Guralnik JM, Simonsick EM, Ferrucci L, Glynn RJ, Berkman LF, Blazer DG, et al. A short physical performance battery assessing lower extremity function: association with self-reported disability and prediction of mortality and nursing home admission. J Gerontol. 1994;49(2):M85-94. DOI: 10.1093/geronj/49.2.m85.

13. Yesavage JA, Brink TL, Rose TL, Lum O, Huang V, Adey M, et al. Development and validation of a Geriatric Depression Screening Scale: A preliminary report. J Psychiatr Res. 19821983;17(1):37-49. DOI: 10.1016/0022-3956(82)90033-4

14. Grupo Estatal de Demencias. Plan Integral de Alzheimer y otras Demencias (2019-2023). Madrid; Ministerio de Sanidad, Consumo y Bienestar Social: 2019.

15. Folstein MF, Folstein SE, McHugh PR. "Mini-mental state": A practical method for grading the cognitive state of patients for the clinician. J Psychiatr Res. 1975;12(3):189-98. DOI: 10.1016/00223956(75)90026-6

16. Cabrera González D, Menéndez Caicoya A, Fernández Sánchez A, Acebal García V, García González JV, Díaz Palacios E, et al. Evaluación de la fiabilidad y validez de una escala de valoración social en el anciano. Aten Primaria.1999;23(7):434-40.

17. D’Hyver de las Deses C. Valoración geriátrica integral. Rev Fac Med (Méx). 2017;60(3):38-54. 